\title{
On groups with linear sci growth
}

\author{
by
}

\section{Louis Funar (Grenoble), Martha Giannoudovardi (Athens) and Daniele Ettore Otera (Vilnius)}

\begin{abstract}
We prove that the semistability growth of hyperbolic groups is linear, which implies that hyperbolic groups which are sci (simply connected at infinity) have linear sci growth. Based on the linearity of the end-depth of finitely presented groups we show that the linear sci is preserved under amalgamated products over finitely generated one-ended groups. Eventually one proves that most non-uniform lattices have linear sci.
\end{abstract}

1. Introduction. The metric spaces $\left(X, d_{X}\right)$ and $\left(Y, d_{Y}\right)$ are quasiisometric if there are constants $\lambda, C$ and maps $f: X \rightarrow Y, g: Y \rightarrow X$ (called $(\lambda, C)$-quasi-isometries) such that

$$
\begin{aligned}
d_{Y}\left(f\left(x_{1}\right), f\left(x_{2}\right)\right) & \leq \lambda d_{X}\left(x_{1}, x_{2}\right)+C, & d_{X}(g f(x), x) & \leq C, \\
d_{X}\left(g\left(y_{1}\right), g\left(y_{2}\right)\right) & \leq \lambda d_{Y}\left(y_{1}, y_{2}\right)+C, \quad & d_{Y}(f g(y), y) & \leq C,
\end{aligned}
$$

for all $x, x_{1}, x_{2} \in X$ and $y, y_{1}, y_{2} \in Y$.

Definition 1.1. A connected locally compact locally simply connected topological space $X$ with $\pi_{1} X=0$ is simply connected at infinity (abbreviated $s c i$, and one also writes $\pi_{1}^{\infty} X=0$ ) if for each compact $k \subseteq X$ there exists a larger compact $k \subseteq K \subseteq X$ such that any closed loop in $X \backslash K$ is null homotopic in $X \backslash k$.

The sci is a fundamental tameness condition for non-compact spaces, as it singles out Euclidean spaces among contractible manifolds, following classical results of Stallings and Siebenmann. The concept of sci of finitely presented groups goes back at least to Siebenmann's thesis [25] and seems to first appear in its present form in Houghton's paper [13] (see also [19, 4]), as follows:

2010 Mathematics Subject Classification: 20F65, 20F67, 20F69, 22E40, 57M50.

Key words and phrases: simple connectivity at infinity, quasi-isometry, end-depth, lattices in Lie groups, amalgamated products. 
Definition 1.2. A finitely presented group $G$ is simply connected at infinity (abbreviated sci) if for some (equivalently any) finite complex $X_{G}$ with $\pi_{1} X_{G}=G$, its universal covering $\widetilde{X_{G}}$ is sci.

The group $\mathbb{Z}^{2}$ is obviously not sci. More interestingly, M. Davis (see e.g. 9]) constructed word hyperbolic groups $G$ (of virtual cohomological dimension $n \geq 4$ by the results of Bestvina and Mess from [1]) which are not sci.

All groups considered here will be finitely presented (unless otherwise explicitly stated), and a system of generators determines a word metric on the group. Although this depends on the chosen generating set, different word metrics are quasi-isometric. In [10] we enhanced the topological sci notion in the case of groups by taking advantage of this metric structure.

Definition 1.3. Let $X$ be a sci non-compact metric space. The sci growth $V_{X}(r)$ (called rate of vanishing of $\pi_{1}^{\infty}$ in [10]) is the infimal $N(r)$ with the property that any loop in the complement of the metric ball $B(N(r))$ of radius $N(r)$ (centered at the identity) bounds a 2-disk outside $B(r)$.

REMARK 1.4. It is easy to construct examples of metric spaces with arbitrarily large $V_{X}$.

It is customary to introduce the following (rough) equivalence relation on real valued functions: the real functions $f$ and $g$ are equivalent, denoted by $f \sim g$, if there exist constants $c_{i}, C_{j}$ for $i=1,2,3$ (with $c_{1}, c_{2}>0$ ) such that:

$$
c_{1} f\left(c_{2} x\right)+c_{3} \leq g(x) \leq C_{1} f\left(C_{2} x\right)+C_{3} \quad \text { for all } x .
$$

It is proved in [10] that the (rough) equivalence class of $V_{X}(r)$ is a quasiisometry invariant. In particular, if a finitely presented group $G$ is sci, then the (rough) equivalence class of the real function $V_{G}=V_{\widetilde{X_{G}}}$ is a quasiisometry invariant of $G$, where $\widetilde{X_{G}}$ is the universal covering space of any finite complex $X_{G}$ with $\pi_{1}\left(X_{G}\right)=G$.

If a finitely presented group $G$ is sci and $V_{G}$ is a linear function, we will say that $G$ has linear sci. In contrast with the abundance of equivalence classes of geometric invariants of finitely presented groups (like group growth, Dehn functions or isodiametric functions), the metric refinements of topological properties seem highly constrained. We already found in [10] that many cocompact lattices in Lie groups and in particular geometric 3 -manifold groups have linear sci. The aim of this paper is to further explore this phenomenon by considerably enlarging the class of groups with linear sci. Our first result is:

THEOREM 1.5. Word hyperbolic groups which are sci have linear sci. 
Let us recall that a group $G$ is one-ended (or 0-connected at infinity) if, for any compact subset $L$ of the Cayley graph $X_{G}$ of $G$, there exists a compact subset $K \supset L$ such that any two points outside $K$ can be joined by a path contained in $X_{G} \backslash L$.

If $H$ is a subgroup of two groups $G_{1}$ and $G_{2}$, the amalgamated product $G_{1} *_{H} G_{2}$ is the quotient of the free product of $G_{1}$ and $G_{2}$ where the copies of $H$ in $G_{1}$ and $G_{2}$ are identified. If $H$ and $K$ are isomorphic subgroups of $G_{1}$, the $H N N$-extension $G_{1} *_{H}$ is the quotient of the free product $G_{1} *\langle t\rangle$, where $H$ is identified with $t^{-1} K t$ and $\langle t\rangle$ denotes the free cyclic group generated by $t$.

We next show that the class of groups with linear sci is closed under amalgamated free products known to preserve the sci.

THEOREM 1.6.

(1) Let $G_{1}$ and $G_{2}$ be one-ended finitely presented groups with linear sci, and $H$ be a finitely generated subgroup of $G_{1}$ and $G_{2}$ with one end. Then the amalgamated free product $G=G_{1} *_{H} G_{2}$ has linear sci.

(2) Let $G_{1}$ be a finitely presented group with linear sci, and $H, K$ be isomorphic finitely generated subgroups with one end. Then the $H N N$ extension $G=G_{1} *_{H}$ has linear sci.

Theorem 1.6 is similar to (but subject to stronger restrictions than) the results obtained by Mihalik and Tschantz [21, 22] in the context of semistability. Notice that the sci is not preserved under amalgamated products over multi-ended subgroups (see [14]).

Previous results of [10] dealt with all cocompact lattices in connected Lie groups but the solvable ones. We now consider non-uniform lattices. Our main result in this direction is:

TheOrem 1.7 .

(1) Let $G$ be a semisimple Lie group for which the associated symmetric space $G / K$ is of dimension $n \geq 4$ and of $\mathbb{R}$-rank greater than or equal to 2 . Let $\Gamma$ be an irreducible, non-uniform lattice in $G$ of $\mathbb{Q}$ rank one. Then $\Gamma$ is sci with linear sci.

(2) Every lattice $\Gamma \subset \mathrm{SO}(n, 1), n \geq 2$, has linear sci.

REMARK 1.8. We believe that the last result also holds for $\mathbb{R}$-rank 1 semisimple Lie groups and for non-uniform lattices of $\mathbb{Q}$-rank $>1$. Further, if moreover strongly polycyclic groups had linear sci, then all lattices in Lie groups (of sufficiently large dimension) would have linear sci.

The results of this paper naturally lead to the question of the existence of sci groups with superlinear sci growth. It seems still unknown whether sci CAT(0) groups have linear sci. 


\section{Proof of Theorem 1.5}

2.1. Preliminaries on hyperbolic groups. Let $(X, d)$ be a geodesic metric space, which in our case will be the Cayley graph of a finitely generated group $G$ endowed with the word metric induced by a finite generating system. Let $\gamma$ be a geodesic path in $X$, possibly infinite. For any $x, y \in \gamma$, we denote by $[x, y]_{\gamma}$ the subpath of $\gamma$ that connects $x$ with $y$. When $\gamma$ is finite, we denote by $\ell(\gamma)$ the length of the path $\gamma$.

A geodesic triangle in $X$ is $\delta$-slim if every side is contained in the $\delta$-neighborhood of the union of the other sides. The group $G$ is $\delta$-hyperbolic, for some $\delta \geq 0$, if all geodesic triangles in $X$ are $\delta$-slim. The group $G$ is $h y$ perbolic if it is $\delta$-hyperbolic for some $\delta \geq 0$. It is well known that hyperbolic groups are finitely presented.

Suppose from now on that $G$ is a $\delta$-hyperbolic group and $X_{G}$ is its Cayley complex associated with a finite presentation $\mathcal{P}=\langle S \mid R\rangle$. We will only consider geodesics within the Cayley graph, that is, the 1-skeleton $X_{G}^{(1)}$ of $X_{G}$. Notice that while the Cayley complex may change when we add words equal to the identity to the relators in $\mathcal{P}$, the Cayley graph remains unchanged.

Bestvina and Mess [1] proved the following crucial fact, which was pointed out by Mihalik:

Proposition 2.1 ([1]). Let $G$ be a hyperbolic one-ended group. There is a constant $c \geq 0$ such that for all $x \in X_{G}$ there exists an infinite geodesic ray issuing from the identity of $G$ which passes within $c$ of $x$.

We say that two geodesic rays are asymptotic if their images in $X_{G}$ are a finite Hausdorff distance apart. This defines an equivalence relation on the collection of geodesic rays in $X_{G}$. The boundary $\partial X_{G}$ of $X_{G}$ is the collection of equivalence classes, under this relation, of geodesic rays in $X_{G}$. Unless otherwise stated, all geodesics considered will be assumed to be unit speed geodesics.

We say that $X_{G}$ satisfies $\operatorname{Rel}(M)$ for some $M>0$ if there exists $L>0$ such that for all $R>0$ and $x, y \in X_{G}$ with $d(x, 1)=d(y, 1)=R$ and $d(x, y) \leq M$ there exists a path of length at most $L$ that connects $x$ and $y$ outside the ball $B(R-c)$, where $c$ is the constant of Proposition 2.1. It will be convenient to say that then $X_{G}$ satisfies $\operatorname{Rel}(M)$ with constant $L$. The significance of $\operatorname{Rel}(M)$ is revealed in the following:

Proposition 2.2 ([1]). Let $G$ be a hyperbolic one-ended group. If $\operatorname{Rel}(M)$ fails for some $M>0$, then $\partial G$ contains a global cut point.

Combining this with a result of Bowditch, Svenson and Swarup [3, 27, 26] which states that $\partial X_{G}$ has no global cut points, we derive that every oneended hyperbolic group satisfies $\operatorname{Rel}(M)$ for any $M>0$. 
2.2. Semistability and simple connectivity at infinity. The aim of this section is to put the sci growth into a more general context. Recall that a ray in a non-compact topological space $X$ is a proper map $\gamma:[0, \infty) \rightarrow X$. As above we consider $X$ to be a connected locally compact locally simply connected topological space. Two rays $\gamma_{1}$ and $\gamma_{2}$ converge to the same end of $X$ if for any compact $C \subset X$ there exists $R$ such that $\gamma_{1}([R, \infty))$ and $\gamma_{2}([R, \infty))$ lie in the same component of $X \backslash C$. The set of rays under this equivalence relation is the same as the set of ends of $X$.

Definition 2.3. An end of $X$ is semistable if any two rays of $X$ converging to this end are properly homotopic. This is equivalent (see e.g. [11]) to the following: for any ray $\gamma$ converging to the end and for any $n \geq 0$, there exists $N \geq n$ such that any loop based on a point of $\gamma$ with image outside the metric ball $B(N)$ of radius $N$ and fixed center can be pushed $($ rel $\gamma$ ) to infinity by a homotopy in $X \backslash B(n)$.

A topological space is semistable if all its ends are semistable. This definition was extended to groups: A finitely presented group $G$ is semistable if for some (equivalently any) finite complex $X_{G}$ with $\pi_{1}\left(X_{G}\right)=G$ its universal covering $\widetilde{X_{G}}$ is semistable.

Many classes of groups are known to be semistable (see e.g. 21, 20, and also [3, 27, 26] for the case of hyperbolic groups) but examples of finitely presented groups which are not semistable are still unknown. There is a well-defined notion of topological fundamental group at infinity associated to a semistable end of a group (see [12]). Now, much as in [10, we consider the following metric refinement of semistability:

Definition 2.4. Let $X$ be a non-compact metric space, $e$ an end of $X$, and $\gamma$ a ray converging to $e$. The semistability growth function $S_{e}(r)$ is the infimal $N(r)$ with the following property: for any $R \geq N$ and any loop $l$ based on $\gamma$ which lies in $X \backslash B(N)$ there exists a homotopy rel $\gamma$ supported in $X \backslash B(r)$ which moves $l$ to a loop in $X \backslash B(R)$.

Set $S_{G}$ for $\sup _{e} S_{e}$, with $e$ running over the set of ends of $\widetilde{X_{G}}$, where $X_{G}$ is a finite complex with fundamental group $G$, whenever this is defined. It is not difficult to see that the equivalence class of $S_{G}$ is a well-defined quasi-isometry invariant of the finitely presented group $G$.

The principal result of this section is the following immediate connection between sci growth and semistability growth:

Proposition 2.5. Assume that $G$ is a finitely presented sci group. Then $V_{G}=S_{G}$.

Proof. For given $r$, as the space $\widetilde{X_{G}}$ is sci there exists some large enough $N(r)$ so that any loop within $\widetilde{X_{G}} \backslash B(N(r))$ bounds a disk outside $B(r)$. 
Let $l$ be a loop not intersecting $B\left(S_{G}(r)\right)$. By the semistability assumption one can homotope $l$ in $\widetilde{X_{G}} \backslash B(r)$ to a loop $l^{\prime}$ lying within $\widetilde{X_{G}} \backslash B(N(r))$. But $l^{\prime}$ bounds a disk outside $B(r)$, and hence $l$ bounds a disk outside $B(r)$. This proves that $V_{G}(r) \leq S_{G}(r)$.

For the reverse inequality let $l$ be a loop based at $\gamma\left(V_{G}(r)+\varepsilon\right.$ ) (for arbitrarily small $\varepsilon$ ) outside $B\left(V_{G}(r)\right)$, where $\gamma$ is a given ray. Then $l$ bounds a disk outside $B(r)$, which yields a nullhomotopy of the based loop $l$ to the base point $p$. We then push $p$ along $\gamma$ as far as we want. This proves that $S_{G}(r) \leq V_{G}(r)$.

It follows that Theorem 1.5 is an immediate consequence of the more general

THEOREM 2.6. The semistability growth of word hyperbolic groups is linear.

2.3. Proof of Theorem 2.6. Consider first the case when $G$ is a oneended hyperbolic group. Let $\delta$ be the hyperbolicity constant for a Cayley complex $X_{G}$ of $G$, and let $c$ be the constant provided by Proposition 2.1. By Proposition 2.2 and the absence of cut points (see [3, 27, 26]), $X_{G}$ satisfies $\operatorname{Rel}(M)$ for some $M>6 c+2 \delta+3$ with constant $L>2 c+4$. Without loss of generality we can assume that $X_{G}$ is associated with a presentation of $G$ that contains as relators all words of length less than $2 L+4 c$ which are equal to the identity in $G$. In this section, unless stated otherwise, the balls we consider will be centered at the identity.

Let $n \in \mathbb{Z}_{+}$and $\gamma$ be a geodesic ray in $X_{G}$ that starts from the identity. We will show that every loop $f$ based at a point $x \in \gamma$ and which lies outside $B(n+2 c)$ can be pushed rel $\gamma$ arbitrarily far away by a homotopy outside $B(n)$.

Let $p, q$ be two adjacent vertices of $f$, and $r=d(p, 1)>n+2 c$. There exist unit speed geodesic rays $\gamma_{0}$ and $\gamma_{1}$ issuing from the identity which pass within $c$ of $p$ and $q$, respectively.

Following [1, Prop. 3.2] we now establish:

Lemma 2.7. For every integer $i \geq 0$, there exists a path $f_{i}$ from $\gamma_{0}(r+i)$ to $\gamma_{1}(r+i)$ such that:

(1) The path $f_{i}$ lies outside $B(r+i-c)$.

(2) For any $j \in\left\{0,1, \ldots, L^{i}\right\}$, there is a unit speed geodesic ray $\gamma_{j / L^{i}}$ issuing from the identity such that

$$
\gamma_{j / L^{i}}(r+i) \in f_{i},
$$

and for $j<L^{i}-1$,

$$
d\left(\gamma_{j / L^{i}}(r+i), \gamma_{(j+1) / L^{i}}(r+i)\right) \leq M .
$$


Proof. We use induction on $i$. From the triangle inequality we obtain $d\left(\gamma_{0}(r), p\right) \leq 2 c$ and $d\left(\gamma_{1}(r), q\right) \leq 2 c+1$, so that $d\left(\gamma_{0}(r), \gamma_{1}(r)\right) \leq 4 c+2<M$. By property $\operatorname{Rel}(M)$, there is a path $f_{0}$ of length at most $L$ which joins $\gamma_{0}(r)$ to $\gamma_{1}(r)$ outside $B(r-c)$, hence the claim holds for $i=0$.

Assume now that the result holds for some $i \geq 0$. By property $\operatorname{Rel}(M)$, for any $j \in\left\{0,1, \ldots, L^{i}-1\right\}$, there is a path $\alpha_{j}:[0, L] \rightarrow X_{G}$, of at most unit speed, that joins $\gamma_{j / L^{i}}(r+i)$ to $\gamma_{(j+1) / L^{i}}(r+i)$ and lies outside $B(r+i-c)$.

Next, for any $k \in\{1, \ldots, L-1\}$, there exists a geodesic ray $\gamma_{k}^{\prime}$ issuing from 1 that passes within $c$ of $\alpha_{j}(k)$. Let $y_{k} \in \gamma_{k}^{\prime}$ be a closest point to $\alpha_{j}(k)$. Then $d\left(y_{k}, \alpha_{j}(k)\right) \leq c$, so that $d\left(y_{k}, 1\right) \geq r+i-2 c$. It follows that there exists $z_{k} \in \gamma_{k}^{\prime}$ with $d\left(z_{k}, 1\right) \geq r+i+1$ and $d\left(z_{k}, y_{k}\right) \leq 2 c+1$. We therefore obtain $d\left(z_{k}, z_{k+1}\right) \leq 6 c+3$. As the geodesic triangle of vertices $1, z_{k}, z_{k+1}$ is $\delta$-slim, we derive that

$$
d\left(\gamma_{k}^{\prime}(r+i+1), \gamma_{k+1}^{\prime}(r+i+1)\right) \leq 6 c+2 \delta+3<M .
$$

Therefore, from $\operatorname{Rel}(M)$ there exists a path of length at most $L$ which lies outside $B(r+i+1-c)$ and joins $\gamma_{k}^{\prime}(r+i+1)$ to $\gamma_{k+1}^{\prime}(r+i+1)$. The concatenation of these paths yields the desired $f_{i+1}$. We set $\gamma_{(j L+k) / L^{i+1}}$ to be the geodesic ray $\gamma_{k}^{\prime}$, thereby concluding the induction step. Notice that

$$
\ell\left(\left[\gamma_{j / L^{i}}(r+i), \gamma_{(j+1) / L^{i}}(r+i)\right]_{f_{i}}\right) \leq L .
$$

Let $P, Q$ be two geodesic arcs joining $p$ and $q$ to $\gamma_{0}(r)$ and $\gamma_{1}(r)$, respectively. For any $N \geq 0$, let $\Phi_{N}(p, q)$ be the closed oriented loop obtained by concatenation of $P, \gamma_{0}([r, r+N]), f_{N}, \gamma_{1}([r, r+N])^{-1}, Q^{-1}$ and the edge $q p$.

Lemma 2.8. The loop $\Phi_{N}(p, q)$ is null homotopic outside $B(n)$ for $N \geq 0$.

Proof. The closed loop $\Phi_{0}(p, q)$ has length at most $L+4 c+2$ and lies outside $B(r-c-1)$. By our hypothesis on the group presentation, this loop bounds a 2-cell $A_{0}$ in the Cayley complex, thereby proving the claim for $N=0$.

Now, for any $i \in\{0,1, \ldots, N-1\}$, let $\Phi_{i}$ be the concatenation of the four paths $\gamma_{0}([r+i, r+i+1]), f_{i+1}, \gamma_{1}([r+i, r+i+1])^{-1}$, and $f_{i}^{-1}$. Then $\Phi_{N}(p, q)$ is homotopic to the product of $\Phi_{0}(p, q)$ and $\Phi_{0} \Phi_{1} \cdots \Phi_{N-1}$. We can further decompose each $\Phi_{i}$ as the composition of loops $\Phi_{i}(j)$ consisting of the concatenation of the following four paths: $\gamma_{j / L^{i}}([r+i, r+i+1])$, $\left[\gamma_{j / L^{i}}(r+i+1), \gamma_{(j+1) / L^{i}}(r+i+1)\right]_{f_{i+1}}, \gamma_{(j+1) / L^{i}}([r+i, r+i+1])^{-1}$, and $\left[\gamma_{j / L^{i}}(r+i), \gamma_{(j+1) / L^{i}}(r+i)\right]_{f_{i}}^{-1}$.

Recall from above that $\ell\left(\left[\gamma_{j / L^{i}}(r+i), \gamma_{(j+1) / L^{i}}(r+i)\right]_{f_{i}}\right) \leq L$. For fixed $i$ and $j$, let $\alpha_{j}:[0, L] \rightarrow X_{G}$ denote a path with image

$$
a_{j}([0, L])=\left[\gamma_{j / L^{i}}(r+i), \gamma_{(j+1) / L^{i}}(r+i)\right]_{f_{i}} .
$$

Then $a_{j}$ has at most unit speed and lies outside $B(r+i-c)$. For any $k$ in $\{0,1, \ldots, L-1\}$, denote by $y_{k}$ a point on the geodesic $\gamma_{k}^{\prime}=\gamma_{(j L+k) / L^{i+1}}$ 
which is closest to $a_{j}(k)$, and by $\beta_{k}$ a geodesic path that joins $\alpha_{j}(k)$ to $y_{k}$. Then $d\left(y_{k}, a_{j}(k)\right) \leq c$ and

$$
r+i-2 c \leq d\left(y_{k}, 1\right) \leq L / 2+r+i+c .
$$

Hence $d\left(y_{k}, \gamma_{k}^{\prime}(r+i+1)\right) \leq L / 2+c+1$ and the path $\left[y_{k}, \gamma_{k}^{\prime}(r+i+1)\right]_{\gamma_{k}^{\prime}}$ lies outside $B(r+i-2 c)$. Therefore, the closed loop obtained by concatenating $\beta_{k}$, $\left[y_{k}, \gamma_{k}^{\prime}(r+i+1)\right]_{\gamma_{k}^{\prime}},\left[\gamma_{k}^{\prime}(r+i+1), \gamma_{k+1}^{\prime}(r+i+1)\right]_{f_{i+1}},\left[\gamma_{k+1}^{\prime}(r+i+1), y_{k+1}\right]_{\gamma_{k+1}^{\prime}}$, $\beta_{k+1}$, and $\left[\alpha_{j}(k), \alpha_{j}(k+1)\right]_{\alpha_{j}}$ has length at most $2 L+4 c-1$ and lies outside $B(r+i-2 c)$. So, it bounds a 2-cell $A_{i, j}(k)$ in the Cayley complex outside $B(n)$. The union $A_{i, j}=\bigcup_{k \in\{0,1, \ldots, L-1\}} A_{i, j}(k)$ of all these 2-cells is the image of a disk filling the loop $\Phi_{i}(j)$ outside $B(n)$. This proves the lemma.

The composition of all loops of the $\Phi_{N}(p, q)$ for $p, q$ successive vertices of the loop $f$ is then freely homotopic within $X_{G} \backslash B(n)$ to a loop consisting of concatenations of paths of the form $f_{N}$ which lie outside $B(r+N-c)$. It follows that for any $N \geq 0$ our initial loop $f \subset X_{G} \backslash B(n+2 c)$ is homotopic rel $\gamma$ to a loop in $X_{G} \backslash B(r+N-c)$ by a homotopy outside $B(n)$.

Eventually, when $G$ is not one-ended, we work on the connected components of $X_{G} \backslash B(n+2 c)$. This proves Theorem 2.6. Another proof can be given following the arguments from [2, 15, 16].

\section{Proof of Theorem 1.6}

3.1. Preliminaries on the end-depth. The sci and its refinement (the sci growth rough equivalence class) are 1-dimensional invariants at infinity for a group $G$, in the sense that they take care of loops and disks. The 0-dimensional analogue of the simple connectivity at infinity is the connectivity at infinity, namely the one-endedness. One could adapt the notion of sci growth to the growth of an end-already considered by Cleary and Riley (see [6]). This leads to the following metric refinement which is the 0-dimensional counterpart of the sci growth:

Definition 3.1. Let $X$ be a one-ended metric space. The end-depth $V_{0}(X)$ of $X$ is the infimal $N(r)$ with the property that any two points which sit outside the ball $B(N(r))$ of radius $N(r)$ can be joined by a path outside $B(r)$.

If $G$ is a finitely generated one-ended group, then the end-depth of $G$ is the (rough) equivalence class of the real function $V_{0, G}=V_{0}\left(X_{G}\right)$, where $X_{G}$ is the Cayley graph of $G$ associated to a finite generating set.

In the same way one can define the end-depth of a specific end of a space or finitely generated group which are not necessarily one-ended. In 23 it is proved that the (rough) equivalence class of $V_{0, G}$ is a well-defined quasi-isometry invariant of one-ended finitely presented groups. Examples of 
groups whose Cayley graphs have dead-ends (i.e. end-depth functions strictly larger than $x+c$ for any $c$ ) were obtained in [6]. Our second result shows that the (rough) equivalence class of the end-depth is not meaningful:

Proposition 3.2. Every finitely generated one-ended group has linear end-depth. More precisely we have the inequality

$$
V_{0}\left(X_{G}\right)(r) \leq 2 r \quad \text { for large enough } r,
$$

where $X_{G}$ denotes the Cayley graph associated to a finite generating set of the group $G$.

We postpone the proof of this proposition to the end of this section.

3.2. End of the proof of Theorem 1.6. Consider the amalgamated product $G=G_{1} *_{H} G_{2}$. Let $X_{1}$ and $X_{2}$ be the standard 2-complexes associated to some finite presentations of $G_{1}$ and $G_{2}$, respectively. Let $S_{H}$ be a finite set of generators of $H$ which are represented by a wedge of loops $Y$ in both $X_{1}$ and $X_{2}$. The space $X$ obtained by attaching $X_{1}$ and $X_{2}$ along $Y$ has fundamental group $G$. Let $C_{H}$ be the Cayley graph of $H$ corresponding to the generators $S_{H}$. The image of $\widetilde{Y}$ in $\widetilde{X}_{i}$ is then homeomorphic to $C_{H}$. Furthermore the universal covering $\widetilde{X}$ is constructed from coset copies of the universal coverings $\widetilde{X}_{1}$ and $\widetilde{X}_{2}$ which are attached along copies of $C_{H}$.

We consider a metric ball $B(r)$ of radius $r$ in $\widetilde{X}$ centered at a fixed point. By compactness, $B(r)$ intersects only finitely many copies of $\widetilde{X}_{1}$ and $\widetilde{X}_{2}$. Since $\widetilde{X}_{1}$ and $\widetilde{X}_{2}$ have linear sci, there exists a constant $c$ such that any loop lying in one copy of either $\widetilde{X}_{1}$ or $\widetilde{X}_{2}$ which is outside $B(c r)$ is contractible by a nullhomotopy outside $B(r)$.

Since the one-ended group $H$ has linear end-depth by Proposition 3.2 , one can find a constant $c_{1}$ such that any two points of a copy of $C_{H}$ lying outside $B\left(c_{1} r\right)$ can be connected by a path within that copy of $C_{H}$ not intersecting $B(c r)$.

The proof that any loop of $\widetilde{X}$ which lies outside $B\left(c_{1} r\right)$ bounds a disk outside $B(r)$ is now standard, following [14]. Any edge loop $L$ starting at $g \in G$ can be written as a word $g a_{1} \cdots a_{n}$, with $a_{i} \in G_{1}$ when $i$ is odd and $a_{i} \in G_{2}$ when $i$ is even, such that the equality $a_{1} \cdots a_{n}=1$ holds in $G$. The structure theorem for amalgamated products implies that there exists some $i$ such that $a_{i} \in H$ (see [18]). Thus the edge subpath $l$ corresponding to the element $a_{i} \in H$ starts and ends in the same copy of $C_{H}$.

We will show that $l$ can be homotoped in $\widetilde{X}$ rel end points into this copy of $C_{H}$. As $L$ lies outside $B\left(c_{1} r\right)$, the end points of $l$ are outside $B\left(c_{1} r\right)$ and by the above argument they can be connected by some path $p$ lying within the same copy of $C_{H}$ and which does not intersect $B(\mathrm{cr})$. The resulting loop $l \cup p$ obtained by gluing together $l$ and $p$ at their common end points is 
therefore contained in one copy of either $\widetilde{X}_{1}$ or $\widetilde{X}_{2}$. Moreover, $l \cup p$ lies in the complement of $B(\mathrm{cr})$. By hypothesis, $G_{i}$ have linear sci, and thus $l \cup p$ can be contracted off $B(r)$. This establishes the claim. The word associated to the path $p$ belongs to $H$ and it can be absorbed into $a_{i-1}$. Thus we obtain a free homotopy of $L$ outside $B(r)$ to a loop $L^{\prime}$ starting at $g$ which corresponds to a word strictly shorter than that of $L$. Then, by induction on $n$, we can decrease the length $n$ until the resulting loop has $n=1$. This proves the first part of Theorem 1.6 .

In order to prove the second part let us recall the HNN construction. If $H$ is a finitely generated subgroup of the finitely presented group $G_{1}$, and $f: H \rightarrow G_{1}$ is a monomorphism from $H$ into $G_{1}$, we set $K=f(H)$. Suppose that $H$ is generated by $a_{1}, \ldots, a_{n}$ and denote by $c_{i}$ the generators $f\left(a_{1}\right), \ldots, f\left(a_{n}\right)$ of $K$. Let

$$
\left\langle b_{1}, \ldots, b_{m}, a_{1}, \ldots, a_{n}, c_{1}, \ldots, c_{n} \mid p_{1}=1, \ldots, p_{k}=1\right\rangle
$$

be a presentation for $G_{1}$. Then the HNN-extension $G=G_{1} *_{H}$ of $G_{1}$ by $f$ has the presentation

$$
\left.\left\langle b_{1}, \ldots, b_{m}, a_{1}, \ldots, a_{n}, c_{1}, \ldots, c_{n}, t\right| p_{i}=1 \text { for } i \leq k, c_{j}=t^{-1} a_{j} t \text { for } j \leq n\right\rangle \text {. }
$$

Consider the 2-complex $X_{1}$ associated to the given presentation of $G_{1}$. It contains two wedges of circles $Y_{H}, Y_{K}$ associated to finite sets of generators of $H$ and $K$. Consider the space $X$ obtained from a copy of $X_{1}$ and a copy of $Y_{H} \times[0,1]$ where $Y_{H} \times\{0\}$ is identified with the copy of $Y_{H}$ in $X_{1}$ and $Y_{H} \times\{1\}$ is identified with the copy of $Y_{K}$ in $X_{1}$ by means of $f$. The universal covering space $\widetilde{X}$ of $X$ can be constructed from coset copies of $\widetilde{X}_{1}$ and $C_{H} \times[0,1]$, where $C_{H}$ denotes the Cayley graph of $H$. As above, $C_{H}$ is the image of $\widetilde{Y}_{H}$ inside $\widetilde{X}_{1}$.

As in the case of an amalgamated product above, the key tool is Britton's lemma giving the structure of an HNN-extension which we state as follows. Suppose we have the equality $g_{0} t^{i_{1}} g_{1} t^{i_{2}} \cdots t^{i_{n}} g_{n}=1$ in $G$, where $g_{k} \in G_{1}$. Then, for some $k$, either $i_{k}>0, i_{k+1}<0$, and $g_{k}$ is in $K$, or else $i_{k}<0$, $i_{k+1}>0$, and $g_{k}$ is in $H$.

We denote by $B(r)$ the metric ball centered at the identity in $\widetilde{X}$. Since each copy of $\widetilde{X}_{1}$ has linear sci, there is $c$ such that any loop in $\widetilde{X}_{1}$ outside the metric ball $B(c r)$ contained in one copy of $\widetilde{X}_{1}$ bounds a disk not intersecting $B(r)$. The metric ball $B(c r)$ intersects only finitely many copies of $\widetilde{Y}_{H} \times[0,1]$. By Proposition 3.2, since $H$ is one-ended, one can choose $c$ large enough such that any two points of one copy of $\widetilde{Y}_{H} \times[0,1]$ which lie outside $B(c r)$ can be joined by a path within this copy, not intersecting $B(r)$.

Let $L$ be an edge loop in $\widetilde{X} \backslash B\left(c^{2} r\right)$. This loop can be represented by a word $g_{0} t^{i_{1}} g_{1} t^{i_{2}} \cdots t^{i_{n}} g_{n}$, where $g_{j} \in G_{1}$ and which is equal to 1 in $G$. If $\sum_{j=1}^{n}\left|i_{j}\right|=0$, then the loop is contained in one copy of $\widetilde{X}_{1}$, and thus 
is contractible off $B(r)$, by hypothesis. When $\sum_{j=1}^{n}\left|i_{j}\right|>0$, let $k$ be the one provided by Britton's lemma in the form stated above. Then the edge path corresponding to the word $t^{\operatorname{sgn}\left(i_{k}\right)} g_{k} t^{\operatorname{sgn}\left(i_{k+1}\right)}$ can be closed in either $C_{H}$ or $C_{K}$ by means of a path with the same end points which does not intersect $B(c r)$. Here $\operatorname{sgn}(i)$ denotes the sign of the non-zero $i$. We obtain a loop lying in a copy of $\widetilde{X}_{1}$ outside $B(c r)$, which can therefore be contracted outside $B(r)$. Thus the loop $L$ is homotopic outside $B(r)$ to a new loop for which the quantity $\sum_{j=1}^{n}\left|i_{j}\right|$ dropped off by two units. The claim follows by induction.

REMARK 3.3. If $G_{i}$ are one-ended sci and $H$ is finitely generated multiended, then $G_{1} *_{H} G_{2}$ is one-ended but not sci according to Jackson [14].

3.3. Proof of Proposition 3.2. The first step is the following lemma:

LEMma 3.4. In a homogeneous locally finite one-ended graph, through any point $p$ passes a discrete geodesic, i.e. an isometrically embedded copy of the integers.

Proof. Since the graph is unbounded, for any $n \in \mathbb{N}$ there exist two vertices at distance $2 n$, joined by a geodesic segment $u_{n}, u_{n-1}, \ldots, u_{-n}$. By homogeneity, we can choose as $u_{0}$ a fixed base point $u_{0}=x_{0}$. Now, this is true for any $n \in \mathbb{N}$, and since the graph is locally finite, there exists, by a compactness argument (e.g. diagonal extraction), the desired geodesic.

Now, Proposition 3.2 follows from the following result:

Proposition 3.5. Let $X$ be a graph as before. Let $r \in \mathbb{N}$ and $K$ be a finite subset of $X$ whose diameter is at most $2 r$. Denote by $C$ a connected component of $X \backslash K$. Then for any point $x$ in $C$, we have the following alternative: either

- $x$ belongs to a geodesic ray (i.e. an embedded copy of the natural numbers) of $X$ within $C$ (and this in particular implies that $C$ is infinite), or else

- the distance from $x$ to $K$ is at most $r$, and $C$ is bounded.

Proof. Let $x$ be a point of $C$. Then, by Lemma 3.4 , there exists a discrete geodesic $\left(u_{n}\right)$ with $n \in \mathbb{Z}$ such that $u_{0}=x$. If $x$ does not belong to any geodesic ray contained in $C$, then one can find $n$ and $m>0$ (both minimal) such that $u_{n}$ and $u_{-m}$ belong to $K$. Since the diameter of $K$ is by hypothesis $\leq 2 r$, one has $m+n \leq 2 r$. This means that the distance $d(x, K)$ from $x$ to $K$ is $\min \{m, n\} \leq(m+n) / 2$. Hence $x$ is within $r$ of $K$.

End of the proof of Proposition 3.2. Whenever $K$ is a ball $B(r)$ of radius $r$ centered at the neutral element of the Cayley graph of the group $G$, Proposition 3.5 implies that any bounded connected component of $X_{G} \backslash B(r)$ is 
included in the ball $B(2 r)$ having the same center and radius $2 r$. In particular, $V_{0}(r) \leq 2 r$.

An alternative proof of Proposition 3.2. Suppose that there is a positive integer $r \geq 2$ such that $V_{0}(r)>2 r$. Then there are a bounded connected component $A$ of $X_{G} \backslash B(r)$ and $a \in A$ such that $d(a, B(r))>r$. As $G$ is one-ended, there is an unbounded connected component $C$ of $X_{G} \backslash B(r)$. Consider the action of $a$ on $X_{G}$ by multiplication. Since $d(a, B(r))>r$, clearly $a B(r)=B(a, r) \subset A$, and there are $x \in A$ and $y \in C$ such that ax, ay $\in B(r)$. Here $B(a, r)$ denotes the metric ball of radius $r$ centered at $a$. Therefore, there is a path $\gamma$ in $B(r)$ that joins $a x$ to $a y$. Then $a^{-1} \gamma$ is a path that joins an element of $A$ to an element of $C$, so it must pass through $B(r)$. Thus, there is $w$ on $\gamma$ so that $a^{-1} w \in B(r)$. This however implies that $w \in B(r) \cap a B(r)$, which is a contradiction. This proves that $V_{0}(r) \leq 2 r$, and hence the end-depth of $G$ is linear.

4. Proof of Theorem 1.7. Let $G$ be a connected, semisimple Lie group with trivial center and without compact factors. Unlike uniform lattices, non-uniform lattices $\Gamma$ in $G$ are not quasi-isometric to the symmetric space $X=G / K$ since they do not act cocompactly on $X$. But one can consider the following construction: chop off every cusp of the quotient $X / \Gamma$ and look at the lifts of each cusp to $X$, giving a $\Gamma$-equivariant union of horoballs in $X$. These horoballs are not disjoint in general; they can be made disjoint by cutting the cusps far enough out precisely when $\Gamma$ has $\mathbb{Q}$-rank one. The resulting space is called the neutered space $X_{0}$ associated to $\Gamma$, and $\Gamma$ acts cocompactly on it. The natural metric on $X_{0}$ is the path metric induced from $X$, given by the infimal length in $X$ of paths contained in $X_{0}$ that join the two points. Then $\Gamma$ endowed with the word metric is quasi-isometric to $X_{0}$ endowed with the path metric. However, sometimes the path metric on $X_{0}$ might be distorted with respect to the original metric on $X$. In order to circumvent this difficulty we first only consider higher rank groups.

Proof of Theorem 1.7. Since $G$ has higher rank, a result due to Lubotzky, Mozes, and Raghunathan (see [17]) states that the embedding of $\Gamma$ endowed with the word metric into $G$ endowed with a left invariant metric is Lipschitz and hence a quasi-isometric embedding. The projection $G \rightarrow G / K$ is a quasiisometry and hence $\Gamma$ is quasi-isometric to an orbit $\Gamma \cdot x_{0} \subset X$ endowed with the restriction of the Riemannian metric $d_{X}$ on $X$. Finally the embedding of an orbit of $\Gamma$ into the neutered space $X_{0}$ is a quasi-isometry when we consider the metric $\left.d_{X}\right|_{X_{0}}$ on $X_{0}$.

By the quasi-isometry invariance of the sci growth, it will be sufficient to prove that $X_{0}$ endowed with the metric $\left.d_{X}\right|_{X_{0}}$ has a linear $V_{X_{0}}$. The metric 
balls $B_{\left(X_{0},\left.d_{X}\right|_{X_{0}}\right)}\left(x_{0}, r\right)$ of radius $r$ centered at $x_{0} \in X_{0}$ for this non-geodesic metric are easy to describe, namely

$$
B_{\left(X_{0},\left.d_{X}\right|_{X_{0}}\right)}\left(x_{0}, r\right)=B_{\left(X, d_{X}\right)}\left(x_{0}, r\right) \cap X_{0}
$$

in terms of the Riemannian metric balls $B_{\left(X, d_{X}\right)}\left(x_{0}, r\right)$.

Now, the neutered space $X_{0}$ is obtained from $X$ by removing a collection of disjoint horoballs, as the $\mathbb{Q}$-rank of $\Gamma$ is at least 2. Then any ball $B_{\left(X, d_{X}\right)}\left(x_{0}, r\right)$ of $X$ intersects only finitely many such horoballs.

This implies that the metric sphere $S_{\left(X_{0},\left.d_{X}\right|_{X_{0}}\right)}\left(x_{0}, r\right) \subset \partial B_{\left(X_{0}, d_{X} \mid x_{0}\right)}\left(x_{0}, r\right)$ is obtained from the usual metric sphere $\partial B_{\left(X, d_{X}\right)}\left(x_{0}, r\right)$ in $X$ by removing from it the intersection with a disjoint union of finitely many horoballs.

We need now a lemma which explains the geometry of such intersections:

Lemma 4.1. Let $X$ be a proper $\mathrm{CAT}(0)$ manifold, $H$ be a horoball, and $B$ be a sphere of $X$. If the center $c$ of $B$ does not belong to $H$, then $B \cap H$ is convex (i.e. topologically a ball).

Proof. Let $f_{c}(x)=d(x, c)$ be the distance function to a fixed point $c \notin H$. Then $f_{c}$ restricted to $H$ has only a critical point in $H$, namely the projection $p(c)$ of $c$ on $H$, where it achieves a non-degenerate minimum. Since $f_{c}$ is proper, the level sets on $H$ retract onto $p(c)$.

From Lemma 4.1 we derive that the metric spheres in $\left(X_{0},\left.d_{X}\right|_{X_{0}}\right)$ are obtained from $S^{n-1}$ by removing finitely many disjoint disks $D^{n-1}$. This means that, whenever the dimension $n$ of $X$ is $n \geq 4$, the metric spheres in $X_{0}$ are simply connected. It follows that $V_{\left(X_{0},\left.d_{X}\right|_{X_{0}}\right)}(r)=r$ is linear and hence $\Gamma$ has linear sci.

For the second part of Theorem 1.7 consider a non-uniform lattice $\Gamma$ in $\mathrm{SO}(n, 1)$. The non-uniform lattice $\Gamma$ acts properly and cocompactly by isometries on $X_{0}=\mathbb{H}^{n} \backslash \mathcal{F}$ where $\mathcal{F}$ is a finite union of disjoint open horoballs. The result does not follow from Lemma 4.1, as the metric on this truncated hyperbolic metric space is the path metric, which is exponentially distorted. Nevertheless this space is CAT(0) (by [5, Cor. 11.28, p. 362] and [24]). Metric balls are therefore homeomorphic to balls and their boundaries are spheres.

In order to understand the topology of the metric spheres it suffices to consider a neighborhood of one horoball $H$. Given $c \in X_{0}$ consider the cone in $\mathbb{H}^{n}$ with vertex $c$ which is tangent to the horoball $H$ along an equidistant $(n-1)$-sphere $S^{n-1}(c) \subset \partial H$. If $p$ belongs to the visible $n$-disk bounded by $S^{n-1}$ on $\partial H$, then the geodesic segments joining $p$ and $c$ for the hyperbolic metric $d_{\mathbb{H}^{n}}$ and the path metric on $X_{0}$ coincide. When $p \in \partial H$ is outside the visible disk then a geodesic segment in the path metric consists of a spherical segment $p q$ joining $p$ to $q \in S^{n-1}(c)$ followed by a geodesic segment $q c$. It follows that metric spheres in the path metric are obtained from a sphere by 
deleting a number of disjoint disks corresponding to visible disks at distance smaller than the radius. For $n \geq 4$ these are simply connected, and this shows that $X_{0}$ with its path metric has linear sci.

5. Other classes of groups with linear sci. Recall that a Coxeter group is a group $W$ with a presentation of the following form:

$$
\left.\left\langle s_{1}, \ldots, s_{n}\right| s_{i}^{2}=1 \text { for } i \in\{1, \ldots, n\},\left(s_{i} s_{j}\right)^{m_{i j}}=1\right\rangle
$$

where $i<j$ ranges over some subset of $\{1, \ldots, n\} \times\{1, \ldots, n\}$ and $m_{i j} \geq 2$. Let $W$ be a Coxeter group.

Proposition 5.1. Coxeter groups which are sci have linear sci.

Proof. The Davis complex $D_{W}$ (see [7]) of a finitely generated Coxeter group $W$ is a CAT(0) cell complex on which $W$ acts cellularly, properly, and with finite quotient. The links of the vertices of $D_{W}$ are all isomorphic to a fixed finite simplicial complex $L$, where $L$ can be described combinatorially in terms of subsets of the generating set of $W$.

It has been proved in 8 that a Coxeter group is sci if and only if its nerve $L$ and all its punctured links $L-\sigma$ are simply connected (where $\sigma$ is any simplex of $L$ ). The boundary of a metric ball in $D_{W}$ is a connected sum of various punctured links $L-\sigma$, and hence it is simply connected.

Now any loop outside the metric ball of radius $r$ can be contracted onto the boundary of the metric ball and there contracted to a point. This implies that $V_{D_{W}}(r)=r$.

The action of the Coxeter group on the Davis complex is not free but has finite stabilizers. Moreover there exists a finite index subgroup which acts freely on the Davis complex. This finite index subgroup is still sci and quasi-isometric to $D_{W}$, and hence by the previous arguments it has linear sci. This implies that $W$ has linear sci.

REMARK 5.2. The same proof shows that a sci right-angled Artin group has linear sci. More generally, Artin groups are semistable and have linear semistability (see [20]).

REMARK 5.3. The connectivity of the punctured links determines the connectivity of $W$ at infinity. However in [9] the authors constructed a CAT(0) cell complex acted on properly and cocompactly by $W$ whose nerve and punctured links are not simply connected, though $W$ is sci. Thus the linear sci is the geometric property of groups which is closest to the "simple connectivity of large spheres".

REMARK 5.4. If $1 \rightarrow H \rightarrow G \rightarrow K \rightarrow 1$ is an exact sequence of finitely presented infinite groups where either $H$ or $K$ has one end, then $G$ has a linear sci growth, by [14]. 
REMARK 5.5. Mihalik and Tschantz [21, 22] have proved that amalgamated products and HNN-extensions of semistable groups over arbitrary finitely generated subgroups are semistable. We do not know whether Theorem 1.6 can be extended to multi-ended subgroups and linear semistability.

Acknowledgements. The authors are indebted to Y. de Cornulier, F. Haglund, P. Papazoglou, V. Poenaru, M. Sapir and A. Valette for useful discussions and advice, and the referee for corrections of both mathematical and historical nature.

The authors acknowledge partial support from Research Funding Program Heracleitus II of the University of Athens and the European Union (M.G.), CMIRA Explora Pro 1200613701 (L.F.) and the European Commission's Marie Curie Intra-European Fellowship and INDAM of Italy (D.O.).

\section{References}

[1] M. Bestvina and G. Mess, The boundary of negatively curved groups, J. Amer. Math. Soc. 4 (1991), 469-481.

[2] M. Bonk and B. Kleiner, Quasi-hyperbolic planes in hyperbolic groups, Proc. Amer. Math. Soc. 133 (2005), 2491-2494.

[3] B. Bowditch, Connectedness properties of limit sets, Trans. Amer. Math. Soc. 35 (1999), 3673-3686.

[4] S. G. Brick, Quasi-isometries and ends of groups, J. Pure Appl. Algebra 86 (1993), $23-33$.

[5] M. R. Bridson and A. Haefliger, Metric Spaces of Non-Positive Curvature, Grundlehren Math. Wiss. 319, Springer, Berlin, 1999.

[6] S. Cleary and T. Riley, A finitely presented group with unbounded dead-end depth, Proc. Amer. Math. Soc. 134 (2006), 343-349; Erratum, ibid. 136 (2008), 2641-2645.

[7] M. W. Davis, Groups generated by reflections and aspherical manifolds not covered by Euclidean space, Ann. of Math. 117 (1983), 293-324.

[8] M. W. Davis and J. Meier, The topology at infinity of Coxeter groups and buildings, Comment. Math. Helv. 77 (2002), 746-766.

[9] M. W. Davis and J. Meier, Reflection groups and CAT(0) complexes with exotic local structures, in: High-Dimensional Manifold Topology, World Sci., River Edge, NJ, 2003, 151-158.

[10] L. Funar and D. E. Otera, A refinement of the simple connectivity at infinity for groups, Arch. Math. (Basel) 81 (2003), 360-368.

[11] R. Geoghegan, Topological Methods in Group Theory, Grad. Texts in Math. 243, Springer, New York, 2008.

[12] R. Geoghegan and M. L. Mihalik, The fundamental group at infinity, Topology 35 (1996), 655-669.

[13] C. H. Houghton, Cohomology and the behaviour at infinity of finitely presented groups, J. London Math. Soc. (2) 15 (1977), 465-471.

[14] B. Jackson, End invariants of amalgamated free products, J. Pure Appl. Algebra 23 (1982), 243-250. 
[15] I. Kapovich and N. Benakli, Boundaries of hyperbolic groups, in: Combinatorial and Geometric Group Theory, Contemp. Math. 296, Amer. Math. Soc., Providence, RI, 2002, 39-93.

[16] B. Kleiner, The asymptotic geometry of negatively curved spaces: uniformization, geometrization and rigidity, in: Proc. Int. Congress of Mathematicians (Madrid, 2006), Vol. II, Eur. Math. Soc., Zürich, 2006, 743-768.

[17] A. Lubotzky, S. Mozes and M. S. Raghunathan, The word and Riemannian metrics on lattices of semisimple groups, Inst. Hautes Études Sci. Publ. Math. 91 (2000), $5-53$.

[18] R. C. Lyndon and P. E. Schupp, Combinatorial Group Theory, Springer, Berlin, 1977.

[19] M. L. Mihalik, Ends of groups with the integers as quotient, J. Pure Appl. Algebra 35 (1985), 305-320.

[20] M. L. Mihalik, Semistability of Artin and Coxeter groups, J. Pure Appl. Algebra 111 (1996), 205-211.

[21] M. L. Mihalik and S. T. Tschantz, Semistability of amalgamated products, HNNextensions, and all one-relator groups, Bull. Amer. Math. Soc. (N.S.) 26 (1992), 131-135.

[22] M. L. Mihalik and S. T. Tschantz, Semistability of amalgamated products and HNNextensions, Mem. Amer. Math. Soc. 98 (1992), no. 471.

[23] D. E. Otera, Some remarks on the ends of groups, Acta Univ. Apulensis Math. Inform. 15 (2008), 133-146.

[24] K. Ruane, CAT(0) boundaries of truncated hyperbolic space, Topology Proc. 29 (2005), 317-331.

[25] L. C. Siebenmann, The obstruction to finding a boundary for an open manifold of dimension greater than five, $\mathrm{PhD}$ thesis, Princeton Univ., 1965, $152 \mathrm{pp}$.

[26] G. A. Swarup, On the cut point conjecture, Electron. Res. Announc. Amer. Math. Soc. 2 (1996), 98-100.

[27] E. L. Swenson, A cut point theorem for CAT(0) groups, J. Differential Geom. 53 (1999), 327-358.

Louis Funar

Institut Fourier

BP 74, UMR 5582, Université Grenoble I

38402 Saint-Martin-d'Hères Cedex, France

E-mail: louis.funar@ujf-grenoble.fr

Daniele Ettore Otera

Institute of Mathematics and Informatics

Vilnius University

Akademijos st. 4

LT-08663, Vilnius, Lithuania

E-mail: daniele.otera@mii.vu.lt

daniele.otera@gmail.com
Martha Giannoudovardi Department of Mathematics University of Athens 15784 Athens, Greece E-mail: marthag@math.uoa.gr martha.giannoudovardi@gmail.com

Received 5 December 2013;

in revised form 11 July 2014 